\title{
LIPSCHITZ CLASSIFICATION OF FUNCTIONS ON A HÖLDER TRIANGLE
}

\author{
L. BIRBRAIR, A. FERNANDES, AND D. PANAZZOLO
}

\begin{abstract}
The problem of semialgebraic Lipschitz classification of quasihomogeneous polynomials on a Hölder triangle is studied. For this problem, the "moduli" are described completely in certain combinatorial terms.
\end{abstract}

\section{$\S 1$. INTRODUCTION}

A fundamental question of singularity theory of functions is the classification of these functions near a singular point up to a change of variables. One can consider continuous changes of variables (topological equivalence) or smooth changes of variables (differential equivalence). The first problem in these directions is the following. Is the classification problem tame? In other words, is the set of equivalence classes countable?

For the polynomial functions of degree less than or equal to some integer $d$, Fukuda [4] proved a finiteness result for topological equivalence: the problem of topological equivalence is tame.

For differential equivalence, it is known that the problem is far from being tame. Lipschitz equivalence [5] and $\mathcal{K}$-Lipschitz equivalence [3] can be regarded as classification problems "between" topological and differential classification. The recent results in this direction are somewhat surprising: the problem of $\mathcal{K}$-Lipschitz equivalence is tame [3] and the problem of Lipschitz equivalence is not [5. $\mathcal{K}$-Lipschitz equivalence is closely related to the usual Lipschitz equivalence. Two functions $f$ and $g$ are said to be $\mathcal{K}$ Lipschitz equivalent if their graphs have the same contact with the hyperplane $y=0$. Henry and Parusinski [5] produced a continuous family of quasihomogeneous polynomials that contains infinitely many Lipschitz nonequivalent germs.

When a classification problem admits such families, we say that the problem has "moduli", i.e., the problem is not tame. The results of [5] motivate the following question: What is the nature of the "moduli" for the problem of Lipschitz classification?

In this paper we describe these moduli for the simplest possible case: quasihomogeneous polynomial functions defined on the so-called Hölder triangle. Note that the problem of Lipschitz classification of functions can also be viewed as a problem of Lipschitz classification of algebraic foliations. An algebraic foliation near a singular point can be decomposed into some elementary pieces: algebraic foliations on Hölder triangles. That is why the classification question on Hölder triangles is a fundamental step. The equivalence relation used in this paper is semialgebraic Lipschitz equivalence. This

2000 Mathematics Subject Classification. Primary 32S15, 32S05.

Key words and phrases. Lipschitz classification, quasihomogeneous polynomials, Hölder triangle, moduli.

The first author was supported by CNPq grant 300985/93-2. The second author was supported by CNPq grant 300393/2005-9, and also by CNPq/FUNCAP/PPP. The third author was supported by CNPq grant 305904/2003-5. 
equivalence became important after the results of Vallete [7] who made a significant improvement of the finiteness theorem of Mostowski [6].

We solve the problem of semialgebraic Lipschitz classification of quasihomogeneous polynomials on a Hölder triangle completely: we present a complete invariant, the socalled multiplicity symbols. This means that the moduli are described completely.

\section{§. BASIC DEFINITIONS}

The Hölder triangle $T_{\beta} \subset \mathbb{R}^{2}$ is a semialgebraic set defined as follows:

$$
T_{\beta}=\left\{(x, y) \in \mathbb{R}^{2}: 0 \leq x, 0 \leq y \leq x^{\beta}\right\},
$$

where $\beta$ is a positive rational number.

A polynomial $F(X, Y)$ is said to be admissible with respect to $T_{\beta}$ if the boundary $\partial T_{\beta}$ is a subset of $\left\{(x, y) \in \mathbb{R}^{2}: F(x, y)=0\right\}$. A polynomial $F(X, Y)$ is reduced in $T_{\beta}$ if $F(X, Y)$ is admissible with respect to $T_{\beta}$ and $F(x, y) \neq 0$ for all $(x, y) \in \operatorname{Int}\left(T_{\beta}\right)$.

Two polynomials $F(X, Y)$ and $G(X, Y)$ admissible with respect to $T_{\beta}$ are said to be $\mathcal{R}$-semialgebraically Lipschitz equivalent on $T_{\beta}$ if there exists a germ of a semialgebraic bi-Lipschitz homeomorphism $\Phi:\left(T_{\beta}, 0\right) \rightarrow\left(T_{\beta}, 0\right)$ such that $F=G \circ \Phi$.

Two polynomials $F(X, Y)$ and $G(X, Y)$, admissible with respect to $T_{\beta}$, are semialgebraically Lipschitz equivalent on $T_{\beta}$ if there exist a germ of a semialgebraic bi-Lipschitz homeomorphism $\Phi:\left(T_{\beta}, 0\right) \rightarrow\left(T_{\beta}, 0\right)$ and a semialgebraic bi-Lipschitz homeomorphism $l:[0, \epsilon) \rightarrow[0, \epsilon)$ such that $l(0)=0$ and $l \circ F=G \circ \Phi$. (We remind the reader that a map is said to be semialgebraic if its graph is a semialgebraic set.)

Let $\beta$ be a positive rational number. We say that a polynomial $F(X, Y)$ is $\beta$-quasihomogeneous of degree $d$ if for all $t \geq 0$ we have

$$
F\left(t x, t^{\beta} y\right)=t^{d} F(x, y), \quad(x, y) \in \mathbb{R}^{2} .
$$

\section{§3. Height FunCtions}

Let $F(X, Y)$ be a $\beta$-quasihomogeneous reduced polynomial on $T_{\beta}$. The height function $f$ associated with $F$ is the restriction of $F$ to the subset of $T_{\beta}$ defined by $\left\{(x, y) \in T_{\beta}\right.$ : $x=1\}$. A height function can be regarded as a polynomial function $f:[0,1] \rightarrow \mathbb{R}$ such that $f(0)=f(1)=0$.

We say that two functions $f, g:[0,1] \rightarrow \mathbb{R}$ are Lipschitz equivalent if there exists a biLipschitz homeomorphism $\phi:[0,1] \rightarrow[0,1]$ and a positive constant $c$ such that $c \cdot f=g \circ \phi$.

Lemma 3.1. Let $f, g:[0,1] \rightarrow \mathbb{R}$ be two polynomial functions. Let $\phi:[0,1] \rightarrow[0,1]$ be a homeomorphism such that $f=g \circ \phi$. If $g$ is a nonconstant function, then $\phi$ is semialgebraic.

Proof. Let

$$
0=t_{0}<t_{1}<\cdots<t_{p}=1
$$

be a partition of the interval $[0,1]$ such that $g$ is monotone on each subinterval $\left[t_{i}, t_{i+1}\right]$. Suppose that $\phi$ is a positive homeomorphism. In this case, let

$$
0=s_{0}<s_{1}<\cdots<s_{p}=1
$$

be a partition of the interval $[0,1]$ such that $\phi\left(s_{i}\right)=t_{i}$. Then, on each subinterval $\left[s_{i}, s_{i+1}\right]$ we have $\phi=g^{-1} \circ f$. Since $f$ and $g$ are polynomials, we see that $g^{-1} \circ f$ is a semialgebraic function and $\phi$ is a semialgebraic function. The case where $\phi$ is a negative homeomorphism is similar. 
Theorem 3.2. Let $\beta>1$ be a rational number. Two $\beta$-quasihomogeneous polynomials reduced in $T_{\beta}$ are $\mathcal{R}$-semialgebraically Lipschitz equivalent if and only if they have the same degree and the corresponding height functions are Lipschitz equivalent. Moreover, these polynomials are semialgebraically Lipschitz equivalent on $T_{\beta}$ if and only if they are $\mathcal{R}$-semialgebraically Lipschitz equivalent on $T_{\beta}$.

Proof. Let $F(X, Y)$ and $G(X, Y)$ be two $\beta$-quasihomogeneous polynomials reduced in $T_{\beta}$ of degree $d$. Suppose that the corresponding height functions $f$ and $g$ are Lipschitz equivalent. By Lemma 3.1 there exists a positive constant $c$ and a semialgebraic biLipschitz homeomorphism $\phi:[0,1] \rightarrow[0,1]$ such that $c \cdot f=g \circ \phi$. Let $\left(x, t x^{\beta}\right)$ be a point in $T_{\beta}$. We define

$$
\Phi\left(x, t x^{\beta}\right)=\left(\lambda x, \phi(t)(\lambda x)^{\beta}\right),
$$

where $\lambda^{d}=c^{-1}$. Clearly, $\Phi$ is semialgebraic. Since $c \cdot f=g \circ \phi$, we have $F=G \circ \Phi$ on $T_{\beta}$. On the other hand, the map inverse to $\Phi$ can be constructed in the same form as (3.1), and we conclude that $\Phi$ is a semialgebraic bi-Lipschitz homeomorphism. We see that $F$ and $G$ are $\mathcal{R}$-semialgebraically Lipschitz equivalent on $T_{\beta}$, and thus, they are semialgebraically Lipschitz equivalent on $T_{\beta}$.

Suppose now that there exists a germ of a semialgebraic bi-Lipschitz homeomorphism $\Phi:\left(T_{\beta}, 0\right) \rightarrow\left(T_{\beta}, 0\right)$ and a semialgebraic bi-Lipschitz homeomorphism $l:[0, \epsilon) \rightarrow[0, \epsilon)$ such that $l(0)=0$ and $l \circ F=G \circ \Phi$. The map $\Phi$ can be written in the form $\Phi(x, y)=$ $\left(\phi_{1}(x, y), \phi_{2}(x, y)\right)$. Two cases are possible.

Case 1. $\Phi(x, 0)=(\varphi(x), 0)$ and $\Phi\left(x, x^{\beta}\right)=\left(\delta(x),[\delta(x)]^{\beta}\right)$.

Case 2. $\Phi(x, 0)=\left(\delta(x),[\delta(x)]^{\beta}\right)$ and $\Phi\left(x, x^{\beta}\right)=(\varphi(x), 0)$.

First, we study Case 1 . Consider $\gamma_{t}(x)=\left(x, t x^{\beta}\right)$, where $t \in[0,1]$.

Proposition 3.3. The germ of the curve $\Phi\left(\gamma_{t}(x)\right)$ at 0 can be presented in the following way:

$$
\Phi\left(\gamma_{t}(x)\right)=\left(\lambda x+r_{1}(t, x),(\lambda x)^{\beta} a(t)+r_{2}(t, x)\right),
$$

where $\lim _{x \rightarrow 0^{+}} \frac{r_{1}(t, x)}{x}=0$ and $\lim _{x \rightarrow 0^{+}} \frac{r_{2}(t, x)}{x^{\beta}}=0$. Moreover, the value $\lambda$ does not depend on the choice of $t$.

Proof. Since $\Phi$ is a bi-Lipschitz semialgebraic homeomorphism, it follows that $\phi_{1}\left(\gamma_{t}(x)\right)=$ $\lambda x+r_{1}(t, x)$ with $\lambda \neq 0$, where $\lim _{x \rightarrow 0^{+}} \frac{r_{1}(t, x)}{x}=0$. Next, since $\Phi$ is Lipschitz, we have a positive constant $K$ such that

$$
\left|\Phi(x, 0)-\Phi\left(x, t x^{\beta}\right)\right| \leq K\left|(x, 0)-\left(x, t x^{\beta}\right)\right|=K t x^{\beta} .
$$

Since $\Phi(x, 0)=\left(\lambda_{0} x+o(x), 0\right)$ and $\beta>1$, we conclude that $\lambda=\lambda_{0}$. On the other hand,

$$
\phi_{2}\left(x, t x^{\beta}\right) \leq\left|\Phi(x, 0)-\Phi\left(x, t x^{\beta}\right)\right| \leq K t x^{\beta} .
$$

Since $\phi_{2}$ is a semialgebraic function, we see that $\phi_{2}\left(\gamma_{t}(x)\right)=a(t)(\lambda x)^{\beta}+o\left(x^{\beta}\right)$. This proves the proposition.

Note that the value $a(t)$ defined in the above proposition can be viewed as a function depending on $t$.

Proposition 3.4. The function a $(t)$ is Lipschitz.

Proof. Consider two curves $\gamma_{t}$ and $\gamma_{s}$. We have

$$
\left|\Phi\left(\gamma_{t}(x)\right)-\Phi\left(\gamma_{s}(x)\right)\right| \leq K|t-s| x^{\beta} .
$$

Therefore,

$$
\left|\phi_{2}\left(\gamma_{t}(x)\right)-\phi_{2}\left(\gamma_{s}(x)\right)\right| \leq K|t-s| x^{\beta} .
$$


But $\phi_{2}\left(\gamma_{t}(x)\right)=a(t)(\lambda)^{\beta} x^{\beta}+o\left(x^{\beta}\right)$. Hence,

$$
\left|a(t)(\lambda)^{\beta} x^{\beta}-a(s)(\lambda)^{\beta} x^{\beta}\right| \leq 2 K|t-s| x^{\beta}
$$

for $x>0$ sufficiently small. Finally,

$$
|a(t)-a(s)| \leq \frac{2 K}{\lambda^{\beta}}|t-s| .
$$

Proposition 3.5. Let $f$ and $g$ be the height functions corresponding to $F$ and $G$. Then, there exists a positive number $c$ such that $c \cdot f=g \circ a$, where a is the function defined above.

Proof. Since $F$ is $\beta$-quasihomogeneous, we have

$$
x^{d_{F}} f(t)=x^{d_{F}} F(1, t)=F\left(x, t x^{\beta}\right),
$$

where $d_{F}$ is the $\beta$-quasihomogeneous degree of $F$. Since $F$ and $G$ are semialgebraically Lipschitz equivalent, Propositions 3.3 and 3.4 yield

$$
\begin{aligned}
l\left(F\left(x, t x^{\beta}\right)\right) & =G\left(\Phi\left(x, t x^{\beta}\right)\right) \\
& =G\left(\lambda x+o(x), a(t)(\lambda x)^{\beta}\right)+o\left(x^{\beta}\right) .
\end{aligned}
$$

For $u$ sufficiently close to 0 , we have $l(u)=\tilde{c} u+o(u)$, because $l$ is a germ of a semialgebraic bi-Lipschitz homeomorphism (here $\tilde{c}$ is a positive constant). Therefore,

$$
\tilde{c} x^{d_{F}} F(1, t)+o\left(x^{d_{F}}\right)=\lambda^{d_{G}} x^{d_{G}} G\left(1+\frac{o(x)}{\lambda x}, a(t)+\frac{o\left(x^{\beta}\right)}{(\lambda x)^{\beta}}\right),
$$

where $d_{G}$ is the $\beta$-quasihomogeneous degree of $G$. Clearly, $d_{F}=d=d_{G}$. Now, passing to the limit as $x \rightarrow 0^{+}$, we obtain

$$
\tilde{c} F(1, t)=\lambda^{d} G(1, a(t)) .
$$

Taking $c=\frac{\tilde{c}}{\lambda^{d}}$, we obtain the proposition.

End of the proof of Theorem 3.2. Since $F$ and $G$ are semialgebraically Lipschitz equivalent, we have $l^{-1} G=F \circ \Phi^{-1}$, where $\Phi^{-1}=\left(\psi_{1}, \psi_{2}\right)$. By Proposition 3.3. $\Phi^{-1}\left(\gamma_{s}(x)\right)$ can be written in the following form:

$$
\Phi^{-1}\left(\gamma_{s}(x)\right)=\left(\tilde{\lambda} x+o(x),(\tilde{\lambda} x)^{\beta} \tilde{a}(s)+o\left(x^{\beta}\right)\right) .
$$

Clearly $\tilde{\lambda}=\lambda^{-1}$.

By Proposition 3.4, $\tilde{a}(s)$ is a Lipschitz function. By Proposition 3.5, we have $c^{-1} g=$ $f \circ \tilde{a}$. Thus, $\tilde{a}=a^{-1}$. This means that $a$ and $\tilde{a}$ are bi-Lipschitz maps.

Using the same arguments, we can prove the result in Case 2.

We have proved that if $F$ and $G$ are semialgebraically Lipschitz equivalent, then the corresponding height functions are Lipschitz equivalent. But we have also proved that the Lipschitz equivalence of height functions implies the $\mathcal{R}$-semialgebraic Lipschitz equivalence of $F$ and $G$. These statements conclude the proof of Theorem 3.2 .

\section{§4. Multiplicity Symbols}

Definition 4.1. A multiplicity symbol $(a, \mu)$ consists of a finite sequence $a_{0}, a_{1}, \ldots, a_{p}$ of real numbers belonging to the interval $[0,1]$ and a function $\mu:\{0,1, \ldots, p\} \rightarrow \mathbb{Z}_{+}$ satisfying the following conditions:

(1) $a_{i}=0$ if and only if $i \in\{0, p\}$;

(2) $a_{i} \neq a_{i+1}$ for all $i=0,1, \ldots, p-1$;

(3) if $a_{i-1}<a_{i}<a_{i+1}$ or $a_{i-1}>a_{i}>a_{i+1}$, then $\mu(i)$ is an even number;

(4) if $a_{i-1}<a_{i}$ and $a_{i}>a_{i+1}$ or $a_{i-1}>a_{i}$ and $a_{i}<a_{i+1}$, then $\mu(i)$ is an odd number; 
(5) $\mu(i)$ can be equal to zero only for $i=0$ or for $i=p$;

(6) there exists $0<i<p$ such that $a_{i}=1$.

Let $f:[0,1] \rightarrow \mathbb{R}$ be a nonnegative analytic function such that $f(0)=f(1)=0$ and $f(t)>0$ for $t \in(0,1)$. A multiplicity symbol associated with $f$ is constructed in the following way. Let $\tilde{f}(t)=\frac{f(t)}{\max (f)}$, where $\max (f)=\max \{f(t): t \in[0,1]\}$, and let $t_{1}, \ldots, t_{p-1}$ be the sequence of all the critical points of $\tilde{f}$ belonging to $(0,1)$. Let $t_{0}=0$ and $t_{p}=1$. Put $a_{i}=\tilde{f}\left(t_{i}\right)$ and let $\mu(i)$ be the multiplicity of $\tilde{f}$ at point $t_{i}$. Define $a=\left(a_{i}\right)_{i}$. Clearly, $(a, \mu)$ is a multiplicity symbol. In this case, $(a, \mu)$ is called the multiplicity symbol corresponding to $f$.

Theorem 4.2. Two polynomial functions $f, g:[0,1] \rightarrow \mathbb{R}$ such that $f(0)=g(0)=$ $f(1)=g(1)=0, f(t)>0$ and $g(t)>0$ for $t \in(0,1)$, are Lipschitz equivalent if and only if the corresponding multiplicity symbols are equal. Moreover, if the multiplicity symbols corresponding to $f$ and $g$ are equal, then $f$ and $g$ are $C^{\infty}$-equivalent.

Proof. Let $(a, \mu)$ be the multiplicity symbol corresponding to $f$ and $g$. Consider the partition

$$
0=t_{0}<t_{1}<\cdots<t_{p-1}<t_{p}=1
$$

of the interval $[0,1]$ such that $t_{1}, \ldots, t_{p-1}$ are the critical points of $f$ on $(0,1)$. Let

$$
0=s_{0}<s_{1}<\cdots<s_{p-1}<s_{p}=1
$$

be a similar partition of $[0,1]$ corresponding to $g$. We define a function $\phi:[0,1] \rightarrow[0,1]$ in the following way:

$$
\left.\phi\right|_{\left[t_{i}, t_{i+1}\right]}=\left.\left.\tilde{g}^{-1}\right|_{\left[a_{i}, a_{i+1}\right]} \circ \tilde{f}\right|_{\left[t_{i}, t_{i+1}\right]} .
$$

Clearly, $\left.\phi\right|_{\left[t_{i}, t_{i+1}\right]}$ is an analytic function. By the classical results on $A_{k}$-singularities, $\phi$ is a $C^{\infty}$ function near each $t_{i}$, because the multiplicity of $\tilde{f}$ at $t_{i}$ is equal to the multiplicity of $\tilde{g}$ at $s_{i}$. By construction, $\phi$ is a monotone function and $\tilde{f}=\tilde{g} \circ \phi$ on $[0,1]$. Hence, $f$ and $g$ are Lipschitz equivalent.

Suppose now that $f$ and $g$ are Lipschitz equivalent, i.e., $c \cdot f=g \circ \phi$ for some positive constant $c$ and a bi-Lipschitz homeomorphism $\phi:[0,1] \rightarrow[0,1]$. It is clear that $\tilde{f}=\tilde{g} \circ \phi$ on $[0,1]$.

Now, we need Lemma 3.1 to conclude that $\phi$ is semialgebraic. Since $\phi$ is a bi-Lipschitz homeomorphism, we see that if $t_{1}, \ldots, t_{p-1}$ are critical points of $\tilde{f}$, then $\phi\left(t_{1}\right), \ldots, \phi\left(t_{p-1}\right)$ are critical points of $\tilde{g}$. Since $\phi$ is a bi-Lipschitz homeomorphism, it follows that for all $t_{i}$, the multiplicity of $\tilde{f}$ at $t_{i}$ is equal to the multiplicity of $\tilde{g}$ at $\phi\left(t_{i}\right)$. Therefore, $f$ and $g$ have the same multiplicity symbol.

Using the results of $\S \S 3$ and 4, we obtain the following classification theorem.

Theorem 4.3. Let $\beta>1$ be a rational number. Two $\beta$-quasihomogeneous polynomials reduced in $T_{\beta}$ are semialgebraically Lipschitz equivalent if and only if they have the same degree and the multiplicity symbols of the corresponding height functions are equal.

\section{REFERENCES}

[1] L. Birbrair, Local bi-Lipschitz classification of 2-dimensional semialgebraic sets, Houston J. Math. 25 (1999), no. 3, 453-472. MR1730886 (2000j:14091)

[2] R. Benedetti and M. Shiota, Finiteness of semialgebraic types of polynomial functions, Math. Z. 208 (1991), no. 4, 589-596. MR.1136477 (92j:14068)

[3] L. Birbrair, J. Costa, A. Fernandes, and M. Ruas, $\mathcal{K}$-bi-Lipschitz equivalence of real function-germs, Proc. Amer. Math. Soc. 135 (2007), no. 4, 1089-1095. MR.2262910 (2007m:58048)

[4] T. Fukuda, Types topologiques des polynômes, Inst. Hautes Études Sci. Publ. Math. No. 46 (1976), 87-106. MR0494152 (58:13080) 
[5] J.-P. Henry and A. Parusinski, Existence of moduli for bi-Lipschitz equivalence of analytic functions, Compositio Math. 136 (2003), no. 2, 217-235. MR.1967391 (2004d:32037)

[6] T. Mostowski, Lipschitz equisingularity, Dissertationes Math. 243 (1985), 46 pp. MR0808226 (87e:32008)

[7] G. Valette, A bilipschitz version of Hardt's theorem, C. R. Math. Acad. Sci. Paris 340 (2005), no. 12, 895-900. MR2152275 (2006a:14097)

Departamento de Matemática, Universidade Federal do Ceará, Av. Mister Hull s/n, CamPus Do PICI, Bloco 914, CEP: 60.455-760 - FortalezA - CE, Brasil

E-mail address: birb@ufc.br

Departamento de Matemática, Universidade Federal do Ceará, Av. Mister Hull s/n, CamPus do PICI, Bloco 914, CEP: 60.455-760 - Fortaleza - CE, Brasil

E-mail address: alexandre.fernandes@ufc.br

Instituto de Matemática e Estatística, Universidade de São Paulo, Rua do Matão 1010, 05508-090 - SÃo PAUlO - SP, BrazIL

E-mail address: dpanazzo@ime.usp.br

Received 16/APR/2007

Originally published in English 